\title{
Therapy with gold nanoparticles and lasers: what really kills the cells?
}

“...any therapy-related study should be based on a well-justified mechanism of laserGNP-cell interactions. Otherwise photothermal nanomedicine may never get from the lab to the clinic."

Two articles were published recently in Nanomedicine: "Enhanced imaging and accelerated photothermalysis of A549 human lung cancer cells by gold nanospheres" by Liu $e t a l$. [1] and "LANTCET: elimination of solid tumor cells with photothermal bubbles generated around clusters of gold nanoparticles" by Hleb et al. [2]. These two articles were published simultaneously and share many similarities: they aim to use gold nanoparticles (GNPs) and laser radiation for the detection and destruction of cancer cells, they use the same model cells (A549) and nanoparticles (gold spheres), and similar in vitro incubation methods of the cells with GNP and similar continuous 633-nm laser radiation is used. Reported results are also comparable: cell damage was observed after GNP and laser treatment. However, closer examination and comparison of these two studies has yielded several significant differences in the experimental methods, models and interpretation of the results. We have presented some of these differences in TABLE 1 assuming that the same cells and the same GNP were studied.

The key idea of photothermal therapy is that GNPs locally convert optical energy into thermal energy through their unique mechanism of plasmon resonance. This idea is the basis behind all published work on GNP-based photothermal methods. The two articles under consideration report almost opposite approaches and results in using 633-nm laser radiation: Liu et al. [1] employs $633 \mathrm{~nm}$ c.w. radiation for inducing NP-mediated damage to the cells, while Hleb et al. [2] uses the same radiation (with possibly lower power) for noninvasive optical monitoring of the same cells and employs different wavelengths of laser radiation $(532 \mathrm{~nm})$ for inducing NP-mediated damage to the cells. We later discuss this apparent contradiction hoping that such discussion may be helpful to the Nanomedicine readership for understanding and optimizing the GNP-based diagnostic and therapeutic methods.

\section{Experimental approach \& results by Lui et al.}

Gold nanoparticles are suggested for cancer optical diagnostics and treatment for their excellent scattering and photothermal properties. Lung cancer cells (A549) were incubated with 40-nm gold spheres conjugated with IgG and were then treated with 633-nm continuous laser radiation. Cell viability was monitored through laser-induced fluorescence of a standard violet dye. The authors have already recently published approximately 20 papers related to the synthesis, modifications and biomedical applications of GNPs.

Targeting of the cells with 40-nm GNP-IgG conjugates: incubation with A549 cells for $24-26$ h at $37^{\circ} \mathrm{C}$

- Experimental results obtained by several independent researchers [3-13] show that IgG conjugates do not bind well with A549 when being applied directly;

- A549 expresses EGF receptor (EGFR) and therefore should be targeted with an anti-EGFR antibody. This was experimentally proved for other types of cancerous cells [3-12,14,15];

- IgG can be used for GNP targeting, but such targeting would require a sandwich method by sequential application to EGFR-positive cells of the primary antibody (anti-EGFR) and then the IgG conjugate. We have verified this method for targeting GNP to cancer cells previously [9-11];

- IgG, during the incubation time of 24-26 h, may kill the cells and therefore is cytotoxic;

- An incubation time of 24-26 h is too long and may cause exocytosis of GNP.

- The described conditions of the incubation are likely to cause the initial endocytosis of GNPs that would remove those GNPs from the cell membrane.

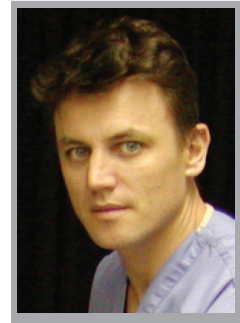

Dmitri Lapotko

A.V. Lykov Heat \& Mass

Transfer Institute, 15 P. Brovka Street, Minsk, 220072, Belarus

Tel.: +375 172842 483;

Fax: +375172842483 ;

E-mail:Id@hmtiac.by 
Table 1. Comparison of the experimental designs for Liu and Hleb.

\section{Subject}

633-nm continuous laser radiation

GNP delivery to the cells

Mechanism of cell damage

\section{Liu et al. [1]}

Used to heat GNP through plasmon resonance optical absorbance and thus damage cancerous cells

IgG-based membrane binding of GNP-IgG conjugates during their incubation with the cells for $24-26 \mathrm{~h}$ at $37^{\circ} \mathrm{C}$

Thermalysis due to laser-induced temperature rise
Hleb et al. [2]

Used to noninvasively probe the cells as does not thermally influence GNP

Nonspecific endocytosis by incubating the cell with bare GNP for $0.5-1 \mathrm{~h}$ at $37^{\circ} \mathrm{C}$

Mechanical damage due to intracellular micro-bubbles

\section{Laser treatment}

Laser treatment of living cells was performed for $58 \mathrm{~s}$ at $633 \mathrm{~nm}\left(0.5-5.0 \mathrm{~mW}, 1 \mathrm{~W} / \mathrm{cm}^{2}\right.$ maximal intensity) and $405 \mathrm{~nm}(0.5 \mathrm{~mW}$, intensity unknown) continuous lasers in the presence of GNP and fluorescent violet dye. Under such conditions there are two heat sources: the dye and GNP. In additiom, there are at least several cell damaging factors, for example:

- Photothermolysis caused by GNPs heated by the laser radiation at $633 \mathrm{~nm}$

- Photothermolysis caused by GNPs heated by the laser radiation at $405 \mathrm{~nm}$

- Photothermolysis caused by the dye heated by $405 \mathrm{~nm}$ laser

- Photochemical and photobiological processes activated with $405 \mathrm{~nm}$ light

- Toxic action of IgG

\section{Results}

The viability of the cells was measured by Liu et al. [1] as the function of time and of 633-nm laser radiation power, and a drop of cell viability down to $8 \%$ has been reported.

The points indicated above cause the main concerns and contradict with the approaches reported by Hleb et al. [2]:

- All five above mechanisms are involved in cell damage, although Liu et al. [1] attribute this mainly to the first point;

- For the first mechanism described in the first one it is well established that the level of cell damage correlates to optical energy/power: it increases with the increase of optical power [4-7,9-11]. However, this does not happen (Figure 5 from $[1,101])$; Lui et al. observed very little dependence of cell death level upon 633-nm power;

- The experience obtained by us and other researchers is that low-power 633-nm laser radiation does not influence cell viability in the presence of spherical GNPs [9-11,16-18]. In addition, several other groups have shown that 633-nm laser radiation does not heat GNPs significantly [19-21];

- Laser- and GNP-induced thermolysis was successfully realized at the wavelengths of the laser radiation that are close to the absorbance peak of spherical GNPs (520$540 \mathrm{~nm}$ ) [3-7,9-11,15,22,23];

- An estimate of the laser- and GNP-induced temperature rise under the conditions that the authors report yields values below $1 \mathrm{~K}$, which cannot cause thermolysis of the cells. For example, the minimal power and exposure time in other similar models of cell photothermolysis [6] were found to be $150 \mathrm{~mW}$ at 4 $\mathrm{min}$ for the laser wavelength of $514 \mathrm{~nm}$ with the corresponding optical absorbance of 40-nm GNPs being 3-4-times higher at this wavelength compared with that at $633 \mathrm{~nm}$.

Therefore, continuous optical excitation at $633 \mathrm{~nm}$ of the spherical GNP (under described parameters) is not likely to cause thermolysis of the cells and can be used for noninvasive optical probing of the cells.

\section{Several other issues that were reported by Liu et al.}

- Liu et al. claim the red-shift of the optical absorbance peak of GNP clusters is relative to the optical absorbance peak wavelength for single GNPs. However, the clusterization of GNP broadens their optical absorbance spectrum but does not shift it significantly into the red region. It can be easily checked by measuring extinction spectrum of the GNP clusters versus that for unclustered GNPs (and was demonstrated in our work). Therefore, GNP clusters still absorb much better at $532 \mathrm{~nm}$ than at $633 \mathrm{~nm}$.

- Imaging methods could be employed to provide a quantitative measure of GNP clusterization in the cells through the analysis of the 
image pixel amplitudes of optical-scattering images. However, this was not done and authors qualitatively concluded about GNP binding to cellular membranes.

- Cell viability was not analyzed after GNPcell interaction and without application of laser radiation. Application of 405-nm laser radiation for the viability measurements is incorrect because this radiation itself does influence cell viability as was found by the authors (see also the mechanisms earlier). Thus, the authors bypassed a very important stage in any cell-nanoparticle model: dark cytotoxicity of GNP conjugates was not measured.

- The viability of the intact cells after laser irradiation (see Figures 4 \& 5 in $[1,101]$ ) was not good (60\%). This compromises the selectivity and safety of GNP photothermal therapy, which are assumed to be the main advantages of this new method over the established ones.
Laser- and GNP-induced photothermolysis was shown by us and other researchers as the method that provides good selectivity, safety and efficacy of cell treatment [4-11,15,22-24]. Those key features of nanoparticle phototherapy significantly depend upon laser-induced physical and biological processes in and around intracellular GNPs. Therefore, any therapy and/or diagnosticrelated study should be based on a well-justified mechanism of laser-GNP-cell interactions. Otherwise photothermal nanomedicine may never get from the lab to the clinic.

\section{Financial \& competing interests disclosure}

The author has no relevant affiliations or financial involvement with any organization or entity with a financial interest in or financial conflict with the subject matter or materials discussed in the manuscript. This includes employment, consultancies, honoraria, stock ownership or options, expert testimony, grants or patents received or pending, or royalties.

No writing assistance was utilized in the production of this manuscript.

\section{Bibliography}

1 Liu X, Lloyd MC, Fedorenko IV, Bapat P, Zhukov T, Huo Q: Enhanced imaging and accelerated photothermalysis of A549 human lung cancer cells by gold nanospheres. Nanomed. 3(5), 617-626 (2008).

2 Hleb EY, Hafner JH, Myers JN et al.: LANTCET: elimination of solid tumor cells with photothermal bubbles generated around clusters of gold nanoparticles. Nanomed. 3(5), 647-667 (2008).

3 Pitsillides CM, Joe EK, Wei X, Anderson RR, Lin CP: Selective cell targeting with light-absorbing microparticles and nanoparticles. Biophys. J. 84(6), 4023-4032 (2003).

4 Huang X, El-Sayed IH, Qian W, El-Sayed MA: Cancer cell imaging and photothermal therapy in the near-infrared region by using gold nanorods, J. Am. Chem. Soc. 128(6), 2115-2120 (2006).

5 El-Sayed IH, Huang X, El-Sayed MA: Selective laser photo-thermal therapy of epithelial carcinoma using anti-EGFR antibody conjugated gold nanoparticles. Cancer Lett. 239 (1), 129-135 (2006).

6 Huang X, Jain PK, El-Sayed IH, El-Sayed MA: Determination of the minimum temperature required for selective photothermal destruction of cancer cells using immunotargeted gold nanoparticles. Photochem. Photobiol. 82(2), 412-417 (2006).
7 El-Sayed IH, Huang X, El-Sayed MA: Surface plasmon resonance scattering and absorption of anti-EGFR antibody conjugated gold nanoparticles in cancer diagnostics: applications in oral cancer. Nano Lett. 5(5), 829-834 (2005).

8 Loo C, Lowery A, Halas NJ, West JL, Drezek R: Immunotargeted nanoshells for integrated cancer imaging and therapy. Nano Lett. 5(4), 709-711 (2005).

9 Lapotko D, Lukianova E, Potapnev M, Aleinikova O, Oraevsky A: Method of laser activated nanothermolysis for elimination of tumor cells. Cancer Lett. 239(1), 36-45 (2006).

10 Lapotko D, Lukianova E, Oraevsky A: Selective laser nano-thermolysis of human leukemia cells with microbubbles generated around clusters of gold nanoparticles. Lasers Surg. Med. 38(6), 631-642 (2006).

11 Lapotko D, Lukianova-Hleb E, Oraevsky A: Clusterization of nanoparticles during their interaction with living cells. Nanomed. 2(2), 241-253 (2007).

12 Sokolov K, Follen M, Aaron J et al.: Real-time vital optical imaging of precancer using anti-epidermal growth factor receptor antibodies conjugated to gold nanoparticles. Cancer Res. 63, 1999-2004 (2003).

13 Pissuwan D, Valenzuela SM, Cortie MB: Therapeutic possibilities of plasmonically heated gold nanoparticles. Trends Biotech. 24(2), 62-67 (2006).
14 Sokolov K, Aaron J, Hsu B et al.: Optical systems for in vivo molecular imaging of cancer. Technol. Cancer Res. Treat. 2(6), 491-504 (2003).

15 Lowery R, Gobin AM, Day ES, Halas NJ, West JL: Immunonanoshells for targeted photothermal ablation of tumor cells. Int. J. Nanomedicine 1(2), 149-154 (2006).

16 Cognet L, Tardin C, Boyer D, Choquet D, Tamarat P, Lounis B: Single metallic nanoparticle imaging for protein detection in cells. Proc. Natl Acad. Sci. USA 100 (20), 11350-11355 (2003).

17 Blad G, Cognet L, Berciaud S et al.: Optical readout of gold nanoparticles-based DNA microarrays without silver enhancement. Biophys. J. 90(1), L13-L15 (2006).

18 Cognet L, Berciaud S, Lasne D, Lounis B: Photothermal methods for single nonluminescent nano-objects. Anal. Chem. 80(7), 2288-2294 (2008).

19 Boyer D, Tamarat P, Maali A, Lounis B, Orrit M: Photothermal imaging of nanometer-sized metal particles among scatterers. Science 297(5584), 1160-1163 (2002).

20 Ortega M, Rodriguez L, Castillo J, Piscitelli V, Fernandez A, Echevarria L: Thermo-optical properties of gold nanoparticles in colloidal systems. J. Opt. A 10, 104024 (2008). 
21 Bertussi B, Natoli J-Y, Commandre M et al.: Photothermal investigation of the laserinduced modification of a single gold nano-particle in a silica film. Opt. Comm. 254(4-6), 299-309 (2005).

22 Gobin A, Lee MH, Halas N, James W, Drezek R, West J: Near-infrared resonant nanoshells for combined optical imaging and photothermal cancer therapy. Nano Lett. 7(7), 1929-1934 (2007).
23 Huang X, Jain P, El-Sayed I, El-Sayed M: Gold nanoparticles: interesting optical properties and recent applications in cancer diagnostics and therapy. Nanomed. 2(5), 681-693 (2007).

24 Tong L, Zhao Y, Huff T, Hansen M, Wei A, Cheng J-X: Gold nanorods mediate tumor cell death by compromising membrane integrity. Avd. Mater. 19(20), 3136-3141 (2007).

\section{Website}

101 Future Medicine.

www.futuremedicine.com/doi/ full/10.2217/17435889.3.5.617 